\title{
DISTAL TRANSFORMATION GROUPS AND FIBRE BUNDLES
}

\author{
BY ROBERT J. ZIMMER
}

Communicated June 20, 1975

1. Introduction. In [1], Furstenberg gave a striking solution to the problem of describing the structure of distal transformation groups on compact metric spaces. $\mathrm{He}$ introduced the notion of an isometric extension of a transformation group, and proceeded to show that every minimal distal transformation group could be built up from a point by taking isometric extensions and inverse limits. While this enables one to answer many questions about distal transformation groups, isometric extensions, in spite of their appealing definition, are not completely understood.

One example of an isometric extension is given by a suitable action in a fibre bundle, with a homogeneous space of a compact group as fibre, and the isometries of the homogeneous space as structure group. These actions are described on pp. 481-482 of [1], and we shall call them homogeneous extensions. Furstenberg [1, p. 482] has raised the question as to whether every isometric extension is a homogeneous extension. In this paper, we announce results concerning the relationship of these two concepts, and implications for the structure of minimal distal actions.

2. Results. Let $G$, a locally compact second countable group, act continuously on the compact metric spaces $X$ and $Y$. Suppose the actions are minimal and distal. Let $p: X \rightarrow Y$ be a continuous, surjective $G$-map, and suppose that $X$ is an isometric extension of $Y[1]$. Let $E(X)$ be the Ellis group of the action of $G$ on $X$. Choose a point $y \in Y$, and let $K=\left\{t \in E(X) \mid t\left(p^{-1}(y)\right)=p^{-1}(y)\right\}$. Then it is known that $K$ is a compact topological group which acts isometrically on $p^{-1}(y)$ [1], [2]. Hence, $K$ acts on $C\left(p^{-1}(y)\right)$, and we let $R$ be the algebra of continuous functions on $p^{-1}(y)$ which are contained in finite-dimensional $K$-invariant subspaces of $C\left(p^{-1}(y)\right)$.

DEFinition. $X$ is called a finitely generated isometric extension of $Y$ if $R$ is a finitely generated algebra.

We remark that this definition is independent of the choice of $y \in Y$. (This follows from the results of $[2, \S 5]$.)

Our results can be summarized in the following theorems.

THEOREM A. Every finitely generated isometric extension is a homogeneous extension.

AMS (MOS) subject classifications (1970). Primary 54H20, 57E05. 
THEOREM B. Every isometric extension is an inverse limit of homogeneous extensions.

THEOREM C. Every minimal distal action of $G$ on a compact metric space can be built up from a point by taking homogeneous extensions and inverse limits.

THEOREM D. There exist isometric extensions that are not homogeneous extensions.

Theorems $\mathbf{B}$ and $\mathrm{C}$ follow in a reasonably straightforward manner from Theorem A. Theorem $\mathrm{C}$ is, of course, a strengthened version of the Furstenberg structure theorem. The proof of Theorem A is rather involved. The starting point for the proof is the work of Knapp [2], who examines the function space properties of isometric extensions. In particular, he shows that function space results which one would expect for homogeneous extensions hold for isometric extensions as well. At the heart of the proof of Theorem $\mathbf{A}$ is a technique similar in spirit to that used in [4, Theorem 4.3] (announced in [3, Theorem A]) in a measure-theoretic context. Full details will appear elsewhere.

\section{REFERENCES}

1. H. Furstenberg, The structure of distal flows, Amer. J. Math. 85 (1963), 477-515. MR 28 \#602.

2. A. W. Knapp, Distal functions on groups, Trans. Amer. Math. Soc. 128 (1967), 140. MR 36 \#5923.

3. R. Zimmer, Extensions of ergodic actions and generalized discrete spectrum, Bull. Amer. Math. Soc. 81 (1975), 633-636.

4. - Extensions of ergodic group actions (submitted).

DEPARTMENT OF MATHEMATICS, HARVARD UNIVERSITY, CAMBRIDGE, MASSACHUSETTS 02138 